\title{
SPOLM2019
}

XIX SIMPÓSIO DE PESQUISA OPERACIONAL ELOGISTICA DA MARINHA RIO DE JANEIRO, RJ, BRASIL - 06 A 08 DE NOVEMBRO DE 2019

\section{ANÁLISE DO ÍNDICE DE INFLAÇÃO BRASILEIRO UTILIZANDO CADEIAS DE MARKOV}

\author{
Marcelo Bolson \\ mhbolson@ucs.br
}

Rafaela Cechin

rafaelacechin@hotmail.com

Fernanda Tomé

ftome1@ucs.br

Rodrigo Biasuz

rbiasuz@ucs.br

Leandro Luis Corso

1lcorso@ucs.br

Universidade de Caxias do Sul (UCS)

Caxias do Sul, Rua Francisco Getúlio Vargas, 1130 - CEP 95070-560

\section{RESUMO}

O controle inflacionário é de fundamental para a economia brasileira. O presente artigo tem por objetivo aplicar Cadeias de Markov para a análise de variação do índice de inflação brasileiro. O estudo teve como base histórica as taxas de inflação no período de janeiro de 2014 a abril de 2019 que, com a aplicação do método, pode-se observar que as maiores ocorrências de variação ficam nas faixas de $0,2 \%$ a $0,39 \%$ e $0,4 \%$ a $0,59 \%$. Ambas obtiveram um valor percentual de 25,4 , resultando em uma probabilidade de $50,8 \%$ de que a inflação permaneça entre as duas faixas citadas. Quanto a taxa negativa, a probabilidade de ocorrência é de $4,8 \%$, indicando que a variação tende a valores positivos. Os maiores tempos de recorrência encontrados são de 63 meses para os intervalos de $-0,2 \%$ a $-0,01 \%$ e $1 \%$ a $1,19 \%$, e o menor de 3,9 meses para a faixa de $0,2 \%$ até $0,59 \%$. O método de Cadeia de Markov apresentou-se eficiente para tal aplicação e o estudo indica que há uma tendência para contínua elevação da inflação no Brasil em um curto espaço de tempo.

Palavra-chave: Pesquisa Operacional; Cadeias de Markov; Inflação; Economia brasileira.

\begin{abstract}
Inflationary control is fundamental to the Brazilian economy. This article aims to apply Markov Chains to the analysis of variation of the Brazilian inflation index. The historical basis of the study is the inflation rates from January 2014 to April 2019, which, according to the application of the method, it is possible to observe that the largest occurrences of variation are in the range of $0.2 \%$ to $0.39 \%$ and $0.4 \%$ to $0.59 \%$. Both
\end{abstract}


obtained a percentage value of 25.4 , resulting in a $50.8 \%$ probability that inflation will remain between the two ranges mentioned. For the negative rate, the probability of occurrence is $4.8 \%$, indicating that the variation tends to positive values. The highest recurrences time found are 63 months for the ranges of $-0.2 \%$ to $-0.01 \%$ and $1 \%$ to $1.19 \%$, and the shortest is 3.9 months for $0.2 \%$ up to $0.59 \%$. The Markov Chain method is efficient for such an application, and the study indicates that there is a tendency for continuous inflation increase in Brazil in a short period.

Keywords: Industrial Engineering; Markov Chain; Inflation; Brazilian Economy.

\section{Como Citar:}

BOLSON, Marcelo; CECHIN, Rafaela; TOMÉ, Fernanda; BIASUZ, Rodrigo; CORSO, Leandro Luis. Análise do índice de inflação brasileiro utilizando Cadeias de Markov. In: SIMPÓSIO DE PESQUISA OPERACIONAL E LOGÍSTICA DA MARINHA, 19., 2019, Rio de Janeiro, RJ. Anais [...]. Rio de Janeiro: Centro de Análises de Sistemas Navais, 2019.

\section{INTRODUÇÃO}

A atual política econômica brasileira, segundo Costa, Campos e Castro Júnior (2016), tem como objetivo o controle inflacionário. A utilização das taxas de juros é a principal ferramenta do Banco Central para estabilização da inflação a longo prazo, mesmo que não tenham resultados determinantes para esse controle. $O$ mesmo autor ressalta a dívida interna do setor público como sendo uma das principais variáveis que impactam a inflação a longo prazo.

A inflação, de acordo com o endereço eletrônico do Banco Central do Brasil, é definida como o aumento dos preços de produtos e serviços, implicando na diminuição do poder de compra da moeda. Estimar as taxas de variação da inflação é de elevada importância para administradores, considerando que as decisões de financiamentos são fortemente ligadas a ela. Em contraponto, não há um padrão de variação da inflação, considerando os diversos fatores que podem influenciá-la. Isso dificulta uma estimativa correta e inviabiliza a utilização de métodos simples de previsão, já que os mesmos se tornam imprecisos e pouco confiáveis (TERRA; PASSADOR, 2012).

O Brasil teve um longo período com elevadas taxas de inflação, sendo que no período após o Plano Real verificou-se um decréscimo das mesmas. A adoção de um sistema de metas também auxiliou no nível e variabilidade da inflação. Há uma série de incertezas relacionadas ao regime inflacionário, as quais podem vir a atingir de forma negativa a produção, consumo e investimentos no país. A partir de regressões com estados markovianos, foi possível perceber que a condução da política econômica pode afetar positiva ou negativamente o nível e variabilidade da inflação (LOPES; SOARES, 2018).

Nesse cenário se destaca a aplicação de Cadeias de Markov, que atuam como um método para determinar a probabilidade de ocorrer um evento futuro, seja ele de qualquer natureza. $\mathrm{O}$ modelo é baseado em uma matriz de transição que sugere que um evento futuro depende apenas do seu estado atual, independente dos eventos passados (HILLIER; LIEBERMAN, 2006). Em razão da sua flexibilidade, modelos autoregressivos de mudança de regime markoviano tem se destacado em aplicações de economia. Um estudo, por exemplo, analisou as taxas de juros no Brasil fazendo o uso de um modelo de Markov; verificou-se que o comportamento dos gastos públicos, bem como as dívidas públicas, pode 
influenciar no aumento ou diminuição de tais taxas brasileiras (MUNIZ; MOREIRA; CAJUEIRO, 2012).

O objetivo deste trabalho é, portanto, calcular a probabilidade de variação do índice referente a inflação no Brasil, utilizando-se Cadeias de Markov, além de obter o tempo de recorrência esperado.

\section{REFERENCIAL TEÓRICO}

Neste capítulo, são abordados Cadeias de Markov e índice de inflação a fim de fundamentar o estudo realizado.

\subsection{CAdeias de Markov}

Verifica-se que há um alto grau de incerteza e probabilidades no comportamento de processos de diferentes áreas. As Cadeias de Markov permitem que engenheiros e outros profissionais consigam descrever e prever tais comportamentos (CARTER; PRICE, 2001).

Processos estocásticos são aqueles que variam ao longo do tempo de forma probabilística (HILLIER et al, 2013). Um processo estocástico é uma Cadeia de Markov quando depende apenas do estado atual. Logo, o processo deve atender a propriedade Markoviana descrita na Equação 1, onde são considerados os momentos $t_{0}, t_{1}, \ldots, t_{n}$, a família de variáveis aleatórias $\left\{C_{t n}\right\}=\left\{x_{1}, x_{2}, \ldots x_{n}\right\}$ (TAHA, 2007; HILLIER et al, 2013).

$P\left(X_{n}=i_{n} \mid X_{n-1}=i_{n-1}, \ldots, X_{0}=i_{0}\right)=P\left(X_{n}=i_{n} \mid X_{n-1}=i_{n-1}\right)$

Essa propriedade markoviana mostra que de fato a probabilidade de um evento futuro é independente dos eventos passados (HILLIER et al, 2013). A matriz de transição $P$ (Equação 2) define uma Cadeia de Markov, onde as probabilidades $p_{i j}$ são estacionárias, não mudam ao longo do tempo e representam a probabilidade de ir do estado $i$ para o estado $j$ em um passo de tempo (CARTER; PRICE, 2001; HILLIER et al, 2013).

$P=\left(\begin{array}{ccccc}p_{11} & p_{12} & p_{13} & \ldots & p_{1 n} \\ p_{21} & p_{22} & p_{23} & \ldots & p_{2 n} \\ \ldots & \ldots & \ldots & \ldots & \ldots \\ p_{n 1} & p_{n 2} & p_{n 3} & \ldots & p_{n n}\end{array}\right)$

A probabilidade inicial de cada vetor pode ser descrita pela notação apresentada na Equação 3, onde cada $p i(0)=P(x 0=i)$ é a probabilidade de que o sistema está inicialmente no estado $i$ (CARTER; PRICE, 2001).

$p(0)=\left(p_{1}(0) p_{2}(0) \ldots p_{n}(0)\right)$

A partir disso, é possível demonstrar o vetor de probabilidade de estado. Como mostrado na Equação 4, $p_{i}(t)$ é a probabilidade de que o processo estará no estado $i$ em qualquer ponto do tempo $t$ se as probabilidades no tempo 0 são conhecidas (CARTER; PRICE, 2001).

$p(t)=\left(p_{1}(t) p_{2}(t) \ldots p_{n}(t)\right)$

Dessa forma, as probabilidades de estado podem ser definidas pela Equação 5: 
$p(0)=p(0) * P^{n}$, para $n=0,1,2, \ldots$

Pode-se verificar que, após um grande número de transições, a probabilidade de o sistema estar em cada estado não depende mais do estado inicial. Em outras palavras, é possível observar que todas as linhas da matriz de transição tendem a um valor idêntico quando ela é elevada a um expoente suficientemente grande (HILLIER; LIEBERMAN, 2006).

\subsection{INFLAÇ̃̃̃O}

Define-se a inflação como uma situação de aumento contínuo e generalizado nos preços, tanto de bens quanto de serviços, dentro de uma economia, resultando na perda de poder aquisitivo da moeda e da população. Em contrapartida, quando se tem uma redução generalizada por um determinado período, é chamada de deflação (GUTIERRES, 2004). Historicamente falando, a inflação assume lugar de destaque a partir da década de 1960, que foi quando se tornou um problema de maior grau, principalmente em países capitalistas avançados (SILVA; MALDONADO FILHO, 2017).

Diversos fatores podem influenciar a dinâmica inflacionária, como as atividades econômicas, expectativas dos agentes, choques de oferta e, até mesmo, o comportamento dos custos de produção. De acordo com os modelos de projeção do Banco Central, a taxa da inflação pode ser dividida em seis componentes: variação cambial, inércia associada a inflação que excede as metas, diferença entre expectativa dos agentes e a meta, choque de oferta, inflação de preços livres e inflação de preços administrados por contratos (STOCKL; MOREIRA; GIUBERTI, 2017).

Silva e Maldonado Filho (2017) abordam as teorias marxista sobre a inflação, sendo elas: a causa mais relevante para a inflação são os conflitos sobre a distribuição de renda, há uma relação próxima entre a inflação e o crescente poder dos monopólios, está relacionada às políticas de intervenção do Estados, e a inflação é explicada pelas grandes diferenças entre os aumentos da oferta e da demanda do dinheiro de crédito.

Em relação à inflação brasileira especificamente, ela não é um fenômeno de demanda, podendo ser explicada por dois componentes principais. O primeiro resulta de um choque internacional dos preços das commodities, enquanto o segundo, se refere à inércia inflacionária. Ou seja, as pressões de custos e o componente inercial explicam grande parte da situação atual da dinâmica inflacionária brasileira (STOCKL; MOREIRA; GIUBERTI, 2017).

Quanto ao controle da inflação, Maycon (2018) explica a dificuldade de uma solução definitiva para esse problema, já que não há incentivo do Estado para a solução de forma sistêmica, apenas como o objetivo de popularidade eleitoral. Além disso, a população como um todo não possui conhecimentos profundos sobre inflação e, em razão disso, acabam delegando a confiabilidade sobre o tema para os governantes. A soma dos dois fatores resulta em péssimas políticas, que não solucionam o problema em questão. A perspectiva para os próximos anos, segundo o Banco Central do Brasil, é inflação de 3,9\%, $4,0 \%$ e $3,75 \%$, respectivamente para os anos de 2019, 2020 e 2021.

\section{MÉTODOS E RESULTADOS}

Para alcançar os objetivos proposto para este estudo, a metodologia divide-se em três etapas: coleta dos dados, elaboração da matriz de transição e análise dos cenários. Tais etapas encontram-se detalhadas em cada uma das subseções a seguir. 


\subsection{COLETA DOS DADOS}

Este estudo utilizou os dados da inflação no Brasil, que é medida pelo Índice Nacional de Preços ao Consumidor Amplo (IPCA), considerando um conjunto de produtos e serviços comercializados, referente ao consumo pessoal da população brasileira. O Instituto Brasileiro de Geografia e Estatística (IBGE) é o responsável por este índice. O IPCA mede a variação do custo de famílias brasileiras com renda mensal de 1 a 40 salários mínimos, contabilizando gastos com alimentação, habitação, vestuário, transporte, saúde, despesas pessoais, educação, e comunicação, de acordo com o endereço eletrônico do Banco Central do Brasil. Os dados são referentes a janeiro de 2014 a abril de 2019 e foram coletados no endereço eletrônico do IBGE. A Figura 1 apresenta o comportamento da variação do IPCA no referido período.

Figura 1 - Variação mensal do IPCA (\%)

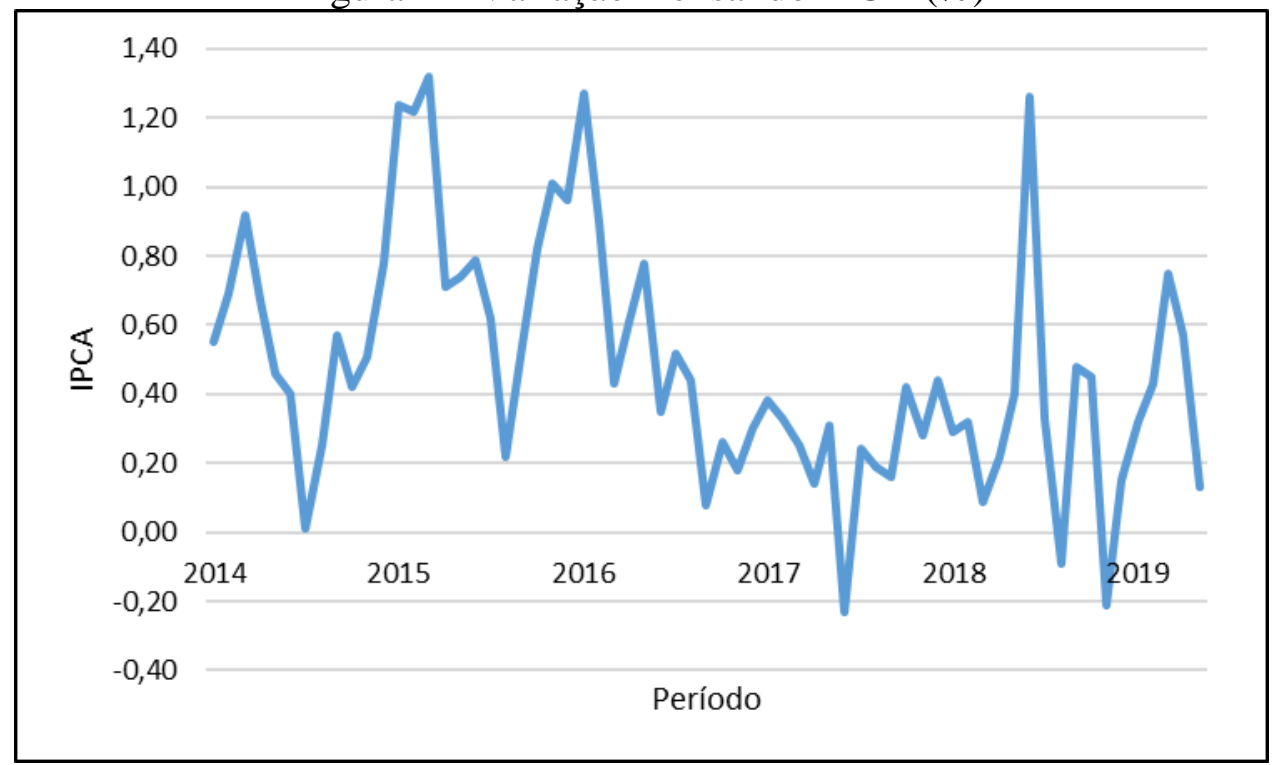

Fonte: Elaborado pelos autores (2019)

Pode-se observar na Figura 1 que a variação do IPCA se comparando os anos de 2014 a 2019 apresentou uma variação de aproximadamente $-0,23$ a 1,32. Verifica-se que em alguns meses entre 2017 e 2019, há valores negativos de IPCA; Isso representa que ocorreu diflação, ou seja, diminuição dos preços. Enquanto, na maior parte do tempo o país apresentou inflação. Tal métrica pode influenciar tanto no mercado financeiro como no poder aquisitivo dos cidadãos.

\subsection{ELABORAÇÃO DA MATRIZ DE TRANSIÇÃO}

Para iniciar os estudos com Cadeia de Markov, foram estabelecidos intervalos de variação, conforme apresentado na Tabela 1. Os dados foram separados de acordo com tais faixas de IPCA.

Tabela 1 - Intervalos de variação

Intervalo de variação

$$
>1,2 \%
$$

de $1 \%$ a $1,19 \%$

de $0,8 \%$ a $0,99 \%$

de $0,6 \%$ a $0,79 \%$ 


\begin{tabular}{c} 
de $0,4 \%$ a $0,59 \%$ \\
de $0,2 \%$ a $0,39 \%$ \\
de $0 \%$ a $0,19 \%$ \\
de $-0,2 \%$ a $-0,01 \%$ \\
$<-0,21 \%$ \\
\hline Fonte: Elaborado pelos autores (2019)
\end{tabular}

Após a definição desses intervalos, foi criada uma matriz de frequência da variação do IPCA, ou seja, da transição de uma faixa para outra. Tais frequências são apresentadas na Figura 2.

Tabela 2 - Matriz de frequência

\begin{tabular}{|c|c|c|c|c|c|c|c|c|c|}
\hline $\begin{array}{c}\text { de } \backslash \\
\text { para }\end{array}$ & $>1,2 \%$ & $\begin{array}{l}1 \% \text { a } \\
1,19 \%\end{array}$ & $\begin{array}{l}\mathbf{0 , 8 \%} \text { a } \\
0,99 \%\end{array}$ & $\begin{array}{l}0,6 \% \text { a } \\
0,79 \%\end{array}$ & $\begin{array}{l}0,4 \% \text { a } \\
0,59 \%\end{array}$ & $\begin{array}{l}0,2 \% \text { a } \\
0,39 \%\end{array}$ & $\begin{array}{l}0 \% \text { a } \\
0,19 \%\end{array}$ & $\begin{array}{c}-0,2 \% \text { a } \\
-0,1 \%\end{array}$ & $\begin{array}{c}< \\
-0,21 \%\end{array}$ \\
\hline$>1,2 \%$ & 2 & 0 & 1 & 1 & 0 & 1 & 0 & 0 & 0 \\
\hline $\begin{array}{l}1 \% \text { a } \\
1,19 \%\end{array}$ & 0 & 0 & 1 & 0 & 0 & 0 & 0 & 0 & 0 \\
\hline $\begin{array}{l}\mathbf{0 , 8 \%} \text { a } \\
0,99 \%\end{array}$ & 1 & 1 & 0 & 1 & 1 & 0 & 0 & 0 & 0 \\
\hline $\begin{array}{c}0,6 \% \text { a } \\
0,79 \%\end{array}$ & 1 & 0 & 1 & 4 & 2 & 2 & 0 & 0 & 0 \\
\hline $\begin{array}{c}0,4 \% \text { a } \\
0,59 \%\end{array}$ & 1 & 0 & 1 & 4 & 5 & 2 & 2 & 0 & 1 \\
\hline $\begin{array}{l}0,2 \% \text { a } \\
0,39 \%\end{array}$ & 0 & 0 & 0 & 0 & 6 & 4 & 4 & 1 & 1 \\
\hline $\begin{array}{c}0 \% \text { a } \\
0,19 \%\end{array}$ & 0 & 0 & 0 & 0 & 1 & 6 & 1 & 0 & 0 \\
\hline $\begin{array}{c}-0,2 \% \text { a } \\
-0,1 \%\end{array}$ & 0 & 0 & 0 & 0 & 1 & 0 & 0 & 0 & 0 \\
\hline $\begin{array}{c}<- \\
0,21 \% \\
\end{array}$ & 0 & 0 & 0 & 0 & 0 & 1 & 1 & 0 & 0 \\
\hline
\end{tabular}

Fonte: Elaborado pelos autores (2019)

A partir da construção da matriz de frequência de transição, tornou-se possível estabelecer o vetor contendo o valor acumulado de cada faixa. A Figura 2 apresenta um histograma com tais valores, onde é mostrado não apenas o número, mas também o percentual cumulativo. 
Figura 2 - Histograma

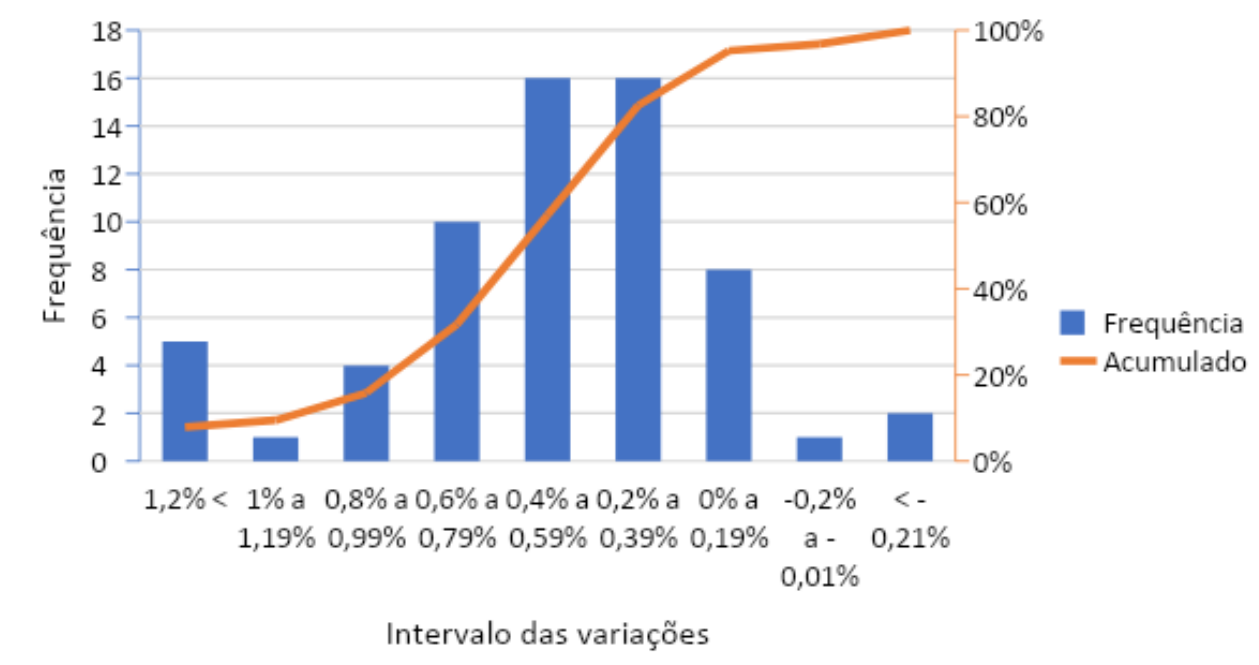

Fonte: Elaborado pelos autores (2019)

Verifica-se na Figura 2 que as frequências de transição variam de 1 a 16 ocorrências; sendo que as faixas centrais de $0,2 \%$ a $0,59 \%$ apresentam os valores mais elevados. A etapa seguinte foi o cálculo da matriz de probabilidade de transição de Markov, apresentada na Tabela 3.

Tabela 3 - Matriz de transição

\begin{tabular}{|c|c|c|c|c|c|c|c|c|c|}
\hline $\begin{array}{l}\text { de } \backslash \\
\text { para }\end{array}$ & $>1,2 \%$ & $\begin{array}{l}1 \% \text { a } \\
1,19 \%\end{array}$ & $\begin{array}{l}\mathbf{0 , 8 \%} \text { a } \\
\mathbf{0 , 9 9 \%}\end{array}$ & $\begin{array}{l}0,6 \% \text { a } \\
0,79 \%\end{array}$ & $\begin{array}{l}0,4 \% \text { a } \\
0,59 \%\end{array}$ & $\begin{array}{l}0,2 \% \text { a } \\
0,39 \%\end{array}$ & $\begin{array}{c}0 \% \text { a } \\
0,19 \%\end{array}$ & $\begin{array}{c}-0,2 \% \text { a } \\
-0,1 \%\end{array}$ & $<-$ \\
\hline$>1,2 \%$ & $40,00 \%$ & $0,00 \%$ & $20,00 \%$ & $20,00 \%$ & $0,00 \%$ & $20,00 \%$ & $0,00 \%$ & $0,00 \%$ & $0,00 \%$ \\
\hline $\begin{array}{l}1 \% \text { a } \\
1,19 \%\end{array}$ & $0,00 \%$ & $0,00 \%$ & $100,00 \%$ & $0,00 \%$ & $0,00 \%$ & $0,00 \%$ & $0,00 \%$ & $0,00 \%$ & $0,00 \%$ \\
\hline $\begin{array}{l}\mathbf{0 , 8 \%} \text { a } \\
\mathbf{0 , 9 9 \%}\end{array}$ & $25,00 \%$ & $25,00 \%$ & $0,00 \%$ & $25,00 \%$ & $25,00 \%$ & $0,00 \%$ & $0,00 \%$ & $0,00 \%$ & $0,00 \%$ \\
\hline $\begin{array}{l}0,6 \% \text { a } \\
0,79 \%\end{array}$ & $10,00 \%$ & $0,00 \%$ & $10,00 \%$ & $40,00 \%$ & $20,00 \%$ & $20,00 \%$ & $0,00 \%$ & $0,00 \%$ & $0,00 \%$ \\
\hline $\begin{array}{l}0,4 \% \text { a } \\
0,59 \%\end{array}$ & $6,25 \%$ & $0,00 \%$ & $6,25 \%$ & $25,00 \%$ & $31,25 \%$ & $12,50 \%$ & $12,50 \%$ & $0,00 \%$ & $6,25 \%$ \\
\hline $\begin{array}{l}0,2 \% \text { a } \\
0,39 \%\end{array}$ & $0,00 \%$ & $0,00 \%$ & $0,00 \%$ & $0,00 \%$ & $37,50 \%$ & $25,00 \%$ & $25,00 \%$ & $6,25 \%$ & $6,25 \%$ \\
\hline $\begin{array}{l}0 \% \text { a } \\
0,19 \%\end{array}$ & $0,00 \%$ & $0,00 \%$ & $0,00 \%$ & $0,00 \%$ & $12,50 \%$ & $75,00 \%$ & $12,50 \%$ & $0,00 \%$ & $0,00 \%$ \\
\hline $\begin{array}{c}-0,2 \% \text { a } \\
-0,1 \%\end{array}$ & $0,00 \%$ & $0,00 \%$ & $0,00 \%$ & $0,00 \%$ & $100,00 \%$ & $0,00 \%$ & $0,00 \%$ & $0,00 \%$ & $0,00 \%$ \\
\hline$<-0,21 \%$ & $0,00 \%$ & $0,00 \%$ & $0,00 \%$ & $0,00 \%$ & $0,00 \%$ & $50,00 \%$ & $50,00 \%$ & $0,00 \%$ & $0,00 \%$ \\
\hline
\end{tabular}

Fonte: Elaborado pelos autores (2019)

\subsection{ANÁLISE DOS CENÁRIOS}

O estudo da análise dos cenários contempla o cálculo da probabilidade do estado estável e do tempo de recorrência esperado. A Tabela 4 apresenta as probabilidades do 
estado estável para cada um dos intervalos em que os dados foram previamente divididos.

\begin{tabular}{cc} 
Tabela $4-$ Probabilidades do estado estável \\
\hline Intervalo & Probabilidade de estado \\
\hline$>1,2 \%$ & $7,90 \%$ \\
de $1 \%$ a $1,19 \%$ & $1,60 \%$ \\
de $0,8 \%$ a $0,99 \%$ & $6,30 \%$ \\
de $0,6 \%$ a $0,79 \%$ & $15,90 \%$ \\
de $0,4 \%$ a $0,59 \%$ & $25,40 \%$ \\
de $0,2 \%$ a $0,39 \%$ & $25,40 \%$ \\
de $0 \%$ a $0,19 \%$ & $12,70 \%$ \\
de $-0,2 \%$ a $-0,01 \%$ & $1,60 \%$ \\
$<-0,21 \%$ & $3,20 \%$ \\
\hline
\end{tabular}

Fonte: Elaborado pelos autores (2019)

É possível notar que os intervalos de $0,2 \%$ a $0,39 \%$ e de $0,4 \%$ a $0,59 \%$ são os que possuem maior probabilidade de estado estável, sendo de $25,4 \%$, o que indica uma tendência de que o valor da inflação se mantenha subindo. Para cada uma destas probabilidades, também foi calculado o tempo de recorrência esperado, conforme mostrado na Tabela 5.

Tabela 5 - Tempo de recorrência esperado

\begin{tabular}{cc}
\hline Estado estável $\pi_{i}$ & Tempo de recorrência esperado \\
& $\mu_{i i}$ \\
\hline$\pi_{0}$ & 12,6 meses \\
$\pi_{1}$ & 63 meses \\
$\pi_{2}$ & 15,7 meses \\
$\pi_{3}$ & 6,3 meses \\
$\pi_{4}$ & 3,9 meses \\
$\pi_{5}$ & 3,9 meses \\
$\pi_{6}$ & 7,9 meses \\
$\pi_{7}$ & 63 meses \\
$\pi_{8}$ & 31,5 meses \\
\hline
\end{tabular}

Fonte: Elaborado pelos autores (2019)

Observa-se que o maior tempo de recorrência para este estudo é de 63 meses, para os intervalos de $-0,2 \%$ a $-0,01 \%$ e de $1 \%$ a $1,19 \%$, enquanto os menores tempos ficam na faixas de 0,2 até $0,59 \%$ de aumento.

\section{CONCLUSÃO}

A inflação gera incertezas para a economia e pode até desestimular novos investimentos no país, prejudicando o crescimento econômico. Ainda, uma alta inflação aumenta o custo da dívida pública. Nesse sentido, indicadores que ajudem a prever o comportamento dessa importante métrica; Junto com suas probabilidades associadas, são fundamentais tanto para possíveis investidores, que têm seus resultados associados a esses índices, quanto para pessoas que precisam gerenciar seus gastos e para governos no planejamento de orçamentos e as estratégias para alocar seus recursos de forma mais precisa.

Nesse cenário, fica evidente a importância de estudos sobre a predição da inflação, e as Cadeias de Markov se apresentam como uma ferramenta que possibilita sinalizar as probabilidades futuras do comportamento desse índice, quando o que se tem disponível é 
apenas o valor histórico do mesmo ao longo do tempo.

A partir do presente estudo, pode-se observar que alguns intervalos apresentaram maior probabilidade de estado estável, representando $25,4 \%$, são eles: $0,2 \%$ a $0,39 \%$ e $0,4 \%$ a $0,59 \%$. Em relação aos tempos de recorrência esperados, observa-se que é de 63 meses para as faixas de $-0,2 \%$ a $-0,01 \%$ e $1 \%$ a $1,19 \%$. Os menores tempos obtidos, de 3,9 meses, localizam-se nos intervalos de $0,2 \%$ até $0,59 \%$. Dessa forma, o estudo indica, com base nos dados históricos, de que há uma tendência para contínua elevação da inflação no Brasil em um curto espaço de tempo.

\section{REFERÊNCIAS BIBLIOGRÁFICAS}

[1] Banco Central do Brasil. Site institucional. https://www.bcb.gov.br/

[2] CARTER, Michael W.; PRICE, Camille C.. Operations research: a practical introduction. Boca Raton: CRC Press, 2001.

[3] COSTA, Cássio Henrique Garcia; CAMPOS, Renato Silvério; CASTRO JÚNIOR, Luiz Gonzaga de. Sensibilidade das Políticas Monetária, Fiscal e Cambial no Combate à Inflação no Brasil. Race - Revista de Administração, Contabilidade e Economia, [s.1.], v. 15, n. 1, p.115-136, mar. 2016. Disponível em: <https://doaj.org/article/0754a754459844929970445b63a6c536?frbrVersion=2> Acesso em: 16 maio 2019.

[4] GUTIERRES, Ana Claudia. O que é inflação?. Revista Eletrônica de Administração, São Paulo, v. 1, n. 1, p.1-3, jun. 2004. Semestral. ISSN 1676-6822. Disponível em: <http://faef.revista.inf.br/imagens_arquivos/arquivos_destaque/TXdwuGboDpj8Fv0_2 013-4-24-14-47-43.pdf> . Acesso em: 16 maio 2019.

[5] LOPES, Luckas Sabioni; SOARES, Thiago Costa. Regimes Inflacionários e Ciclos Econômicos: A Experiência Brasileira Pós-Plano Real. Rev. Bras. Econ., Rio de Janeiro, v. 72, n. 4, p. 410-428, Dec. 2018 . Disponível em: $<$ http://www.scielo.br/scielo.php?script=sci_arttext\&pid=S003471402018000400410\&lng=en\&nrm=iso >. Acesso em: 16 maio 2019.

[6] HILLIER, Frederick S.; LIEBERMAN, Gerald J. Introdução a pesquisa operacional. 8. ed. São Paulo: Mcgraw Hill, 2006.

[7] IBGE. Site institucional. https://www.ibge.gov.br/

[8] MUNIZ, Fabiano Jose; MOREIRA, Tito Belchior S.; CAJUEIRO, Daniel Oliveira. Determinantes da taxa de juros no Brasil: Uma abordagem não-linear usando modelos de Markov-switching. Economia e Desenvolvimento, Recife (PE), v. 11, n. 2, p. 7-40, 2012. Disponível em:

<http://periodicos.ufpb.br/index.php/economia/article/view/16113>. Acesso em: 16 maio 2019. 
[9] MAYCON, David. Como a moeda e a inflação moldam os rumos de nosso desenvolvimento econômico. Mises: Interdisciplinary Journal of Philosophy, Law and Economics, [s.1.], v. 6, n. 2, p.1-6, ago. 2018. Disponível em: <https://doaj.org/article/7af6304d71574e4e9f8e716599c4f0cf>. Acesso em: 15 maio 2019.

[10] SILVA, Giliad de Souza; MALDONADO FILHO, Eduardo Augusto de Lima. Teorias marxistas da inflação: uma revisão crítica. Nova Economia, [s.1.], v. 27, n. 2, p.21-49, ago. 2017. Disponível em:

$<$ http://www.scielo.br/scielo.php?frbrVersion=2\&script=sci_arttext\&pid=S010363512017000200021\&lng=en\&tlng=en>. Acesso em: 15 maio 2019.

[11] STOCKL, Marcos; MOREIRA, Ricardo Ramalhete; GIUBERTI, Ana Carolina. O impacto das commodities sobre a dinâmica da inflação no Brasil e o papel amortecedor do câmbio: evidências para o CRB Índex e Índice de Commodities Brasil. Nova Economia, [s.1.], v. 27, n. 1, p.173-207, abr. 2017. Disponível em: <http://www.scielo.br/scielo.php?frbrVersion=2\&script=sci_arttext\&pid=S010363512017000100173\&lng=en\&tlng=en>. Acesso em: 15 maio 2019.

[12] TAHA, Hamdy A.. Operations Research: an introduction. 8. ed. Upper Saddle River: Pearson Prentice Hall, 2007.

[13] TERRA, Leonardo Augusto Amaral; PASSADOR, João Luiz. Redes neurais artificiais na previsão da inflação: aplicação como ferramenta de apoio à análise de decisões financeiras em organizações de pequeno porte. Ram. Revista de Administração Mackenzie, [s.1.], v. 13, n. 1, p.68-86, fev. 2012. 